\title{
GAMIFICACIÓN EN LA EDUCACIÓN SUPERIOR
}

\section{GAMIFICATION IN HIGHER EDUCATION}

\author{
María Alejandrina Nivela-Cornejo', Omar Efrén Otero- Agreda ${ }^{2}$, Edgar Freddy Morales- \\ Caguana $^{3}$
}

\section{1,2,3 - Universidad de Guayaquil, Guayaquil, Ecuador}

1. Email: maria.nivelac@ug.edu.ec ORCID: https://orcid.org/0000-0002-0356-7243

2. Email: omar.oteroa@ug.edu.ec ORCID: https://orcid.org/0000-0003-0822-6509

3. Email: edgar.moralesc@ug.edu.ec ORCID: https://orcid.org/0000-0002-6977-814X

Recibido: 11/05/2021 Aceptado: 23/07/2021

Para Citar: Nivela-Cornejo, M. A., Otero- Agreda, O. E., \& Morales-Caguana, E. F. (2021). Gamificación en la educación superior. Revista Publicando, 8(31), 165-176. https://doi.org/10.51528/rp.vol8.id2242

\begin{abstract}
Resumen:
La sociedad del conocimiento y la tecnología han traído consigo nuevos escenarios, por esta razón los docentes necesitan explorar nuevas estrategias y recursos para aumentar la motivación y el compromiso de los estudiantes. El objetivo de este trabajo es determinar el impacto, los beneficios del uso de la gamificación y su aplicación en el contexto educativo. Se realizó una investigación cuantitativa con diseño descriptivo. La población fue de 50 estudiantes de pregrado de la Universidad Guayaquil, Facultad de Filosofía, del módulo de Computación. Se elaboró, validó y aplicó un cuestionario de 8 preguntas; 4 con escala de Likert con 5 opciones de respuesta, 2 dicotómicas y 2 abiertas. Se diseñó y aplicó un tratamiento basado en una estrategia de gamificación, durante tres momentos. Como resultados se obtuvo que el $90 \%$ de los estudiantes consideró útil la estrategia, el $80 \%$ opinó que era motivante, el $64 \%$ expresó convenientes los premios y el $84 \%$ de ellos la calificó como muy buena. En conclusión, se validó la efectividad de la estrategia de gamificación en educación usando puntos y premios; sin embargo, es indispensable para mejorar la experiencia de juego incluir las recomendaciones de los estudiantes. Algo que para ellos es fundamental es no hacer examen final.
\end{abstract}

Palabras clave: gamificación, educación, motivación, juego.

\begin{abstract}
:
The society of knowledge and technology have brought new scenarios, for this reason teachers need to explore new strategies and resources to increase the motivation and commitment of students. The objective of this paper is to determine the impact and benefits of the use of gamification and its application in the educational context. A quantitative research was carried out with a descriptive design. The population was 50 undergraduate students from the Guayaquil University, Faculty of Philosophy, from the Computation module. A questionnaire of 8 questions was elaborated, validated and applied; 4 with Likert scale with 5 response options, 2 dichotomous and 2 open. A treatment based on a gamification strategy was designed and applied for three moments. As a result, it was obtained that $90 \%$ of the students considered the strategy useful, $80 \%$ thought it was motivating, $64 \%$ expressed the prizes convenient and $84 \%$ of them rated it as very good. In conclusion, the effectiveness of the gamification strategy in education was validated using points and prizes. However, in order to improve the gaming experience, it is essential to include the recommendations of the students. Something that is essential for them is not to take a final exam.
\end{abstract}

Keywords: gamification, education, motivation, game. 


\section{INTRODUCCIÓN}

$\mathrm{E}$ xiste una necesidad constante de actualizar los métodos educativos, para mejorar la calidad de la educación, esto depende de los contenidos que se imparten, por ello cada vez es más frecuente recurrir a elementos como las TIC y aplicaciones lúdicas que apoyen el proceso. Además, ha quedado constancia de que los estudiantes alcanzan un gran nivel de compromiso cuando se encuentran motivados, incluso prefiriendo seguir con la actividad lúdica a dar por finalizada la clase (Fernández; Olmos y Alegre, 2016). Dentro de estas nuevas demandas surgen nuevas estrategias docentes. En este artículo nos centramos en la gamificación en la educación o gamificación educativa (Marín, 2016).

Esta tendencia, aparece recogida en el informe Horizon Report: 2014 K-12 (Johnson et al., 2014), cuyas recomendaciones se plantean en un plazo de dos a tres años en referencia al aprendizaje basado en juegos y la gamificación como estrategia didáctica, integrando aspectos de la dinámica del juego en contextos no lúdicos que ayuden a potenciar la motivación de los estudiantes, así como otros valores positivos que son usuales en la mayoría de los juegos que se utilizan actualmente para el aprendizaje.

En la mayor parte de las disciplinas los juegos están orientados al objetivo de aprendizaje teniendo fuertes componentes sociales y plantean simulaciones de algún tipo de experiencia del mundo real que los estudiantes encuentran relevante para sus vidas. Como se desprende del informe, la acogida del juego en el mundo académico está haciendo que los desarrolladores respondan con juegos expresamente diseñados para apoyar el aprendizaje inmersivo y experiencial.

La revisión de la literatura se completa con un análisis documental de determinadas experiencias gamificadas, con el fin de estudiar los tres elementos intervinientes según Werbach y Hunter (2012), que vienen a confirmar los resultados planteados por los autores, las dinámicas, las mecánicas y los componentes, aportando, en base a los resultados, conclusiones relevantes mencionados y el uso que en las experiencias seleccionadas se hace de ellos. En base a los planteamientos expuestos, este trabajo está guiado por las siguientes preguntas de investigación: 1 . ¿Qué beneficios aporta el uso de la gamificación en educación y su aplicación en el contexto educativo? 2. ¿En qué medida se confirman los elementos que intervienen en la gamificación según Werbach y Hunter (2012) en las investigaciones seleccionadas en el estudio? por tal motivo, el objetivo de este trabajo es determinar el impacto, los beneficios del uso de la gamificación y su aplicación en el contexto educativo.

\section{REFERENTES TEÓRICOS}

Desde hace años se han introducido aspectos lúdicos en todos los espacios de nuestra vida (Escribano, 2013). Así, se han producido intentos de gamificar actividades en sectores muy diferentes (educación, empresas, recursos humanos, entre otros). En este sentido, encontramos ejemplos de gamificación en muy diferentes ámbitos.

La gamificación se basa en el uso de elementos del diseño de videojuegos en contextos que no son de juego para hacer que un producto, servicio o 
aplicación sea más divertido, atractivo y motivador (Deterding, 2011). Por su parte Zichermann y Cunningham (2011), añade que, mediante la introducción de mecánicas y planteamientos de los juegos, se busca involucrar a los usuarios. Así pues, Burke (2012) plantea la gamificación como el uso de diseños y técnicas propias de los juegos en contextos no lúdicos con el fin de desarrollar habilidades y comportamientos de desarrollo. En este contexto, nuestro planteamiento de gamificación hace referencia a la aplicación de mecánicas de juego a ámbitos que no son propiamente de juego, con el fin de estimular y motivar tanto la competencia como la cooperación entre jugadores (Kapp, 2012, 2016).

De forma mayoritaria, los autores coinciden en señalar la gamificación como un factor fundamental para aumentar la motivación de los

Tabla 1

Elementos de la estrategia de gamificación implementada.

\section{ELEMENTOS DE LA ESTRATEGIA DESCRIPCIÓN}

Objetivos educativos

Comportamientos

Jugadores (rasgos y características) usuarios. Motivar es despertar la pasión y el entusiasmo de las personas para contribuir con sus capacidades y talentos a la misión colectiva (Deterding, 2011; Deterding et al., 2011). Así pues, si se quieren utilizar técnicas de gamificación, se necesita conocer las claves de la motivación para diseñar juegos que enganchen a los distintos tipos de jugadores como veremos más adelante (Valderrama, 2015). De este modo, las técnicas de gamificación están irrumpiendo con fuerza en las organizaciones con el fin de potenciar la motivación y compromiso de empleados y clientes. Los ámbitos de uso van desde la innovación, el marketing, la gestión del talento y el aprendizaje. La Tabla 1 presenta los elementos de la estrategia de gamificación implementada de acuerdo con los aportes de Werbach y Hunter (2012).
- Motivar a los estudiantes a participar activamente en clase

- Dinamizar el desarrollo de los procesos de aprendizaje.

- Mejorar algunas conductas negativas recurrentes de los estudiantes

- Inasistencia

- Falta de participación en clase

- Incumplimiento en la entrega de trabajos

- Baja calidad en la entrega de trabajo

Nativos digitales (Prensky, 2003), jóvenes entre los 16 y 21 años que usan constantemente la tecnología (dispositivos móviles). Además, desean obtener calificaciones altas para mantener promedio de notas superior a 4.0 en la universidad 
- Inicio del semestre: presentación del juego y sus mecánicas

- En cada clase: realización de actividades para la asignación de puntos

- Al finalizar el corte académico: canje de puntos de los estudiantes por los premios definidos

Diversión

Para cada contenido temático se desarrollaron actividades dinámicas (Video quiz, quiz, sopa de letras, crucigrama, entre otros.) en la plataforma Kahoot y Educaplay.

Recursos seguimiento de canje, asignación de puntos

Fuente: elaboración propia de acuerdo con los aportes de Werbach y Hunter (2012)

\section{MATERIALES Y MÉTODOS}

A continuación, se describen métodos, participantes, instrumentos, diseño y procedimiento de la estrategia de gamificación.

\section{Método}

Para determinar la valoración de los estudiantes de la estrategia de gamificación el enfoque de la investigación fue cuantitativo y el diseño descriptivo, permitiendo el registro ordenado y metódico de recolección de datos de acuerdo con las variables establecidas (Briones, 2002).

\section{Participantes}

La estrategia de gamificación contó con la participación de 50 estudiantes de pregrado de la Universidad de Guayaquil, Facultad de Filosofía en los módulos de Computación Dos. De acuerdo con Prensky (2003) nativos digitales con edades que oscilan entre los 16 hasta los 21 años. El mayor porcentaje de estudiantes fue del programa de Literatura con un 20\% (10), la tabla 2 muestra la relación de programas académicos y el número de estudiantes participantes de la estrategia. Respecto a la distribución de sexo el 70\% (35) eran mujeres y el 30\% (15) hombres.

Tabla 2

Estudiantes de la Facultad por programas de gamificación.

\begin{tabular}{lc}
\hline PROGRAMA ACADÉMICO & CANTIDAD DE ESTUDIANTES \\
\hline Literatura & 10 \\
Básico & 9 \\
Químico Biólogo & 9 \\
Historia & 8 \\
Comercio exterior & 7 \\
Arte & 7 \\
\hline
\end{tabular}

Fuente: Elaborado por autores. 
Se elaboró, validó y aplicó un cuestionario para conocer la valoración de los estudiantes respecto a la estrategia utilizada. El instrumento tuvo un total de 8 preguntas; 4 con escala de Likert con 5 opciones de respuesta ( $0=$ Nada; $1=$ Poco; $2=$ Algo; 3=bastante y 4=mucho), 2 dicotómicas, 2 abiertas para conocer lo opinión sobre los premios y la aplicación utilizada en la estrategia de gamificación (Tabla 3).

Tabla 3

Preguntas del instrumento por tipo.

PREGUNTA
TIPO DE

PREGUNTA

1. ¿Considera que la estrategia de gamificación utilizada favorece el desarrollo de los contenidos temáticos de las clases?

Dicotómica

2. ¿Cómo evalúa la estrategia de gamificación utilizada en clases?

Escala Likert

3. ¿Considera que este tipo de estrategias se deben usar en todas las clases de la Universidad para favorecer el aprendizaje y motivación de los estudiantes?

Dicotómica

4. ¿Cómo evalúa la escala de premios utilizada?

Escala Likert

5. ¿Cómo evalúa la aplicación ClassDojo usada en clase para el registro de puntos y asistencias?

6. ¿Qué otros premios y/o incentivos considera que se deben tener en cuenta? Abierta

7. ¿Cómo evalúa la aplicación ClassDojo usada en clases para el registro de puntos y asistencias?

8.¿Qué observaciones tiene acerca de las funciones de la aplicación ClassDojo usada en clases para el registro de puntos?

Escala Likert

Escala Likert

Abierta

Fuente: Elaborado por autores.

\section{Diseño y procedimiento de la estrategia}

La estrategia de gamificación se realizó en 2 grupos (grupo 1, 25 estudiantes; grupo 2, 25 estudiantes) de la asignatura "Computación Dos" de las carreras de la Facultad de Filosofía, Universidad de Guayaquil. La implementación se realizó en 3 momentos (Ramírez, 2014; K. Werbach y Hunter, 2012), de la siguiente forma.

Primero, al inicio del semestre se presentaron las características de interacción, dinámicas y mecánicas del juego, haciendo énfasis en la forma de acumular puntos como asistencia a todas las clases -2 puntos- (asignados al final de cada corte académicos para estudiantes sin fallas en la asignatura); participación -1 punto- (sujeto a la coherencia y pertinencia de la respuesta y/o intervención de cada estudiante en clase), entregas -1 punto- (estudiantes que entregan primero sus trabajos), calidad en las trabajos presentados -2 puntos- (para trabajos con nota de 5). Además, se socializaron premios y escala de canje (Tabla 4 ). 
Tabla 4

Escala de puntos y premios de la estrategia

\begin{tabular}{ll}
\hline PUNTOS & PREMIOS \\
\hline 4 & Un atraso \\
6 & $5(0,5)$ décimas en la nota más baja \\
8 & Borra 1 falta (Una hora de inasistencia a clase) \\
10 & 1 punto en la nota más baja \\
12 & Borra 2 faltas (Dos horas de inasistencia a clase) \\
14 & Borra 3 faltas (Tres horas de inasistencia a clase) \\
\hline
\end{tabular}

Fuente: Elaborado por autores.

Segundo, se presentó a los jugadores de forma inmediata reportes sobre las habilidades (estudiantes) la plataforma de registro y (positivas y negativas) de sus hijos (ClassDojo, seguimiento ClassDojo (http://classdojo.com), 2017).

una herramienta creada en 2011 con el objetivo de transformar la educación mediante la conexión de docentes, estudiantes y padres de familias. La aplicación permite que los docentes creen espacios de interacción (aulas) personalizadas con las habilidades y refuerzos para cada grupo.

Por su parte, los estudiantes cuentan con un usuario de acceso y consulta de avances, debilidades y comunicación con el docente. Además, cada padre de familia recibe una cuenta en la que puede interactuar con el docente y recibir

Para el desarrollo de la estrategia cada estudiante instaló en su dispositivo móvil la aplicación y realizó el registro con el código asignado por el profesor. También, se crearon las habilidades con sus respectivos puntos en la plataforma (Figura 1). Por otra parte, al iniciar cada clase el profesor presentaba en la pantalla el estado de puntos para motivar extrínsecamente a los estudiantes a conseguir más puntos en las actividades que se iban a desarrollar en clase (Ramírez, 2014).

Figura 1

Habilidades de la estrategia creadas en ClassDojo

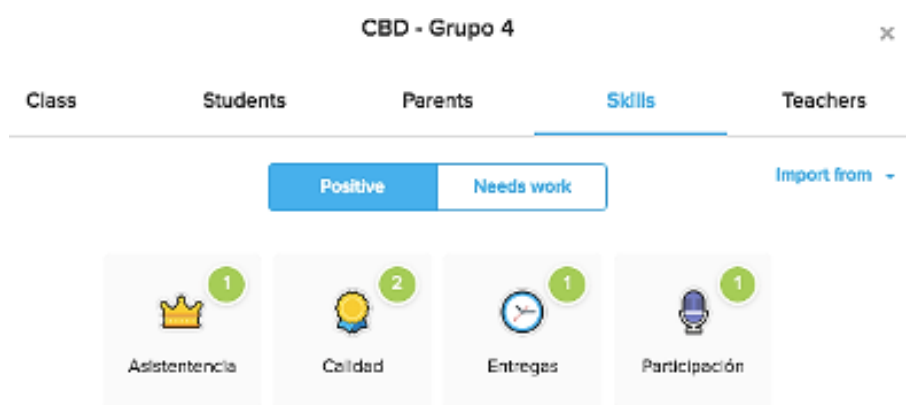

Fuente: Elaborado por autores. 


\section{RESULTADOS Y DISCUSIÓN}

Se presentan los resultados de determinar el impacto, los beneficios del uso de la gamificación y su aplicación en el contexto educativo. En lo referente a la utilidad de la estrategia para el desarrollo de contenidos temáticos en clase (Figura 2), el 90\% de los estudiantes considera que si es útil mientras que el $10 \%$ considera que no. Con relación a la valoración de la utilidad de la estrategia, la mayoría de los estudiantes (20 mujeres ( $57 \%$ de ellas) y 9 hombres (60\% de ellos)) opinó que la valoró mucho (Figura 3).

Al indagar en los estudiantes la motivación generada por la estrategia para el desarrollo de los contenidos temáticos de clase, el 80\% indicó que sí es motivante y el $20 \%$ que no (Figura 4). De acuerdo con estos resultados, se confirma que este tipo de estrategias propicia un entorno favorable para motivar el aprendizaje de los estudiantes en el aula (Dicheva, 2015).

Figura 2

Utilidad de la estrategia de gamificación para el desarrollo de contenidos temáticos en la clase por los estudiantes.

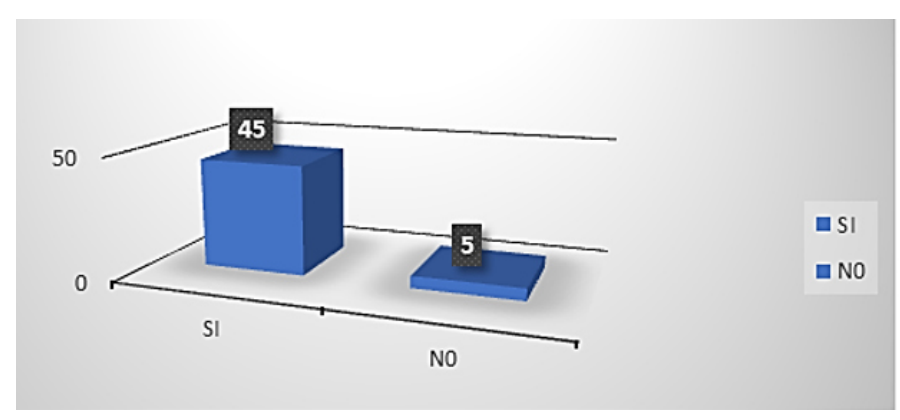

Fuente: Elaborado por autores.

\section{Figura 3}

Distribución de frecuencias sobre la valoración de la utilidad de la estrategia de gamificación por parte de los estudiantes

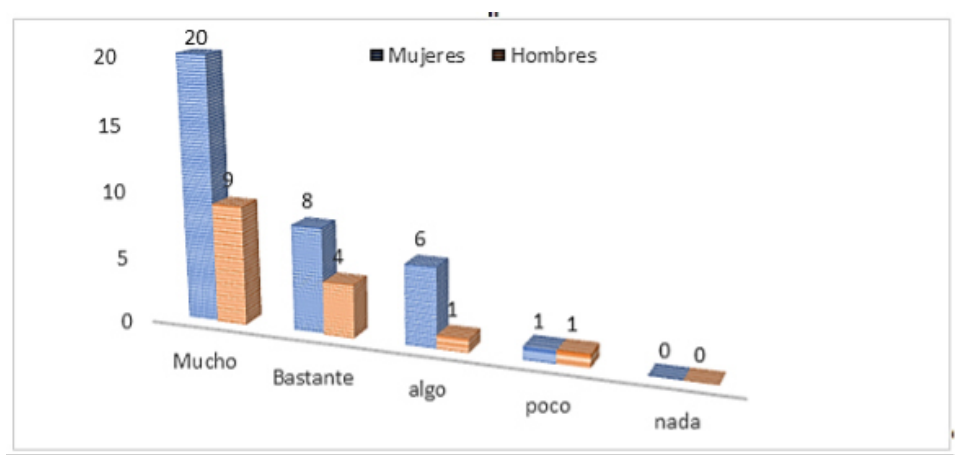

Fuente: Elaborado por autores. 
Figura 4

Motivación de la estrategia de gamificación para el desarrollo de contenidos temáticos en la clase por los estudiantes

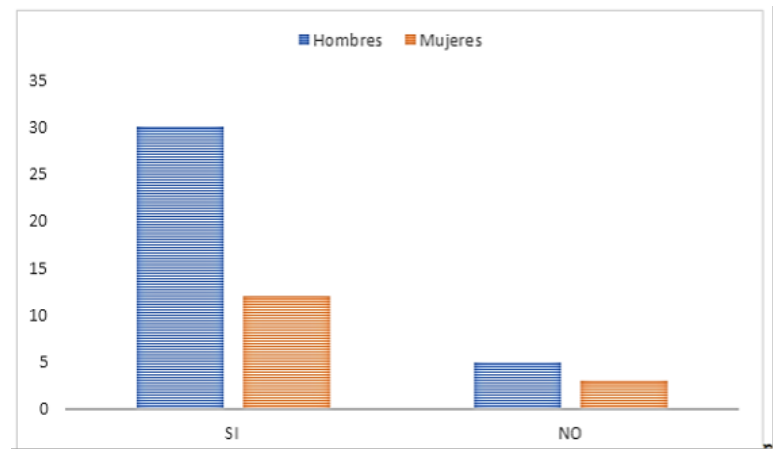

Fuente: Elaborado por autores.

Otro de los aspectos que los estudiantes evaluaron fue la pertinencia de los premios, en este sentido, la mayoría de los estudiantes
(64\%), consideró la conveniencia de los premios dentro de la estrategia como mucho (Figura 5).

\section{Figura 5}

Distribución de frecuencias sobre la pertenencia de los premios de la estrategia de gamificación por parte de los estudiantes.

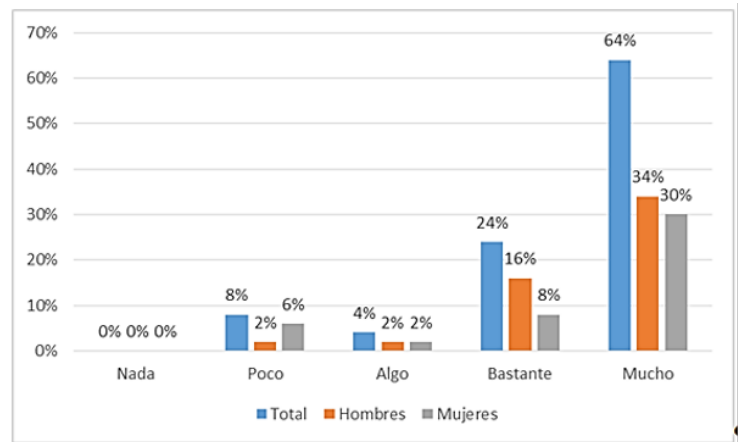

Fuente: Elaborado por autores.

De acuerdo con lo anterior, se preguntó a los estudiantes qué otros premios y/o incentivos podrían ser parte de la estrategia de gamificación. La mayor cantidad de respuestas se centró en aumentar las opciones de canje por calificaciones, además, proponen la eliminación del parcial final y ayudas en el proyecto de aula (Tabla 5). 
Tabla 5

Premios sugeridos por los estudiantes para la estrategia de gamificación.

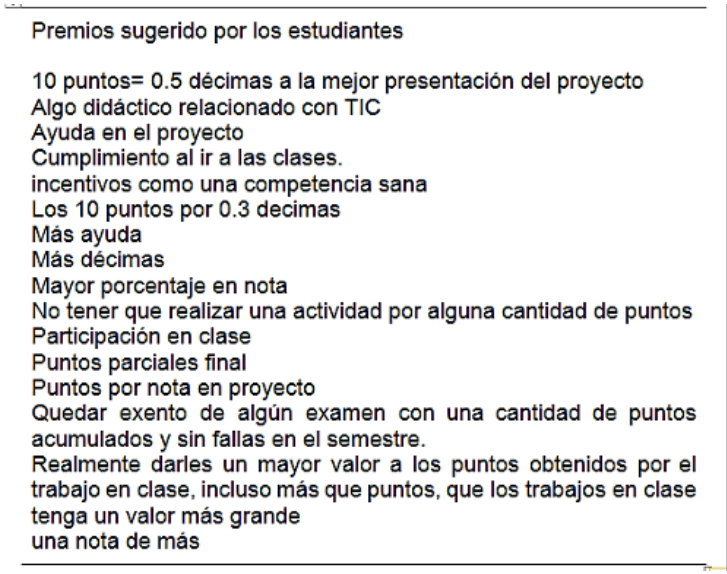

Fuente: Elaborado por autores.

Finalmente, se pidió a los estudiantes que herramientas de gestión académica como registro evaluaran la aplicación ClassDojo, al respecto, el de asistencia, comunicación con padres, entre $84 \%$ de los estudiantes la calificó como bastante y muy buena. Aunque la plataforma cuenta con

otras. En la estrategia implementada sólo se usó para el registro de puntos (Figura 6).

\section{Figura 6}

Distribución de frecuencias sobre la valoración de los estudiantes de la aplicación usada en la estrategia de gamificación ClassDojo

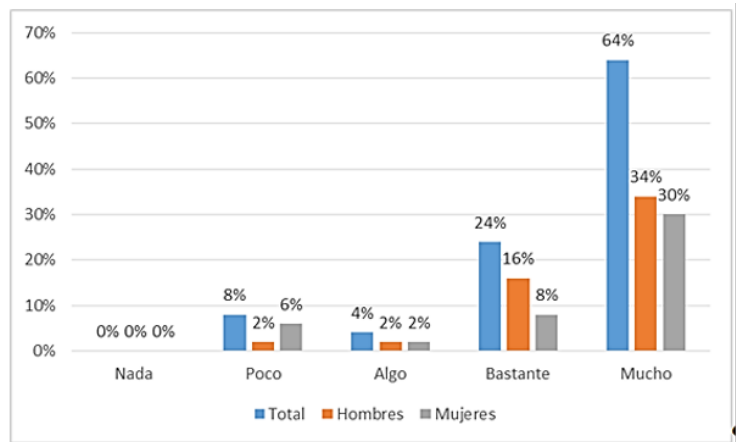

Fuente: Elaborado por autores. 


\section{DISCUSIÓN}

El nivel de valoración de la estrategia es alto $90 \%$, la evaluación de premio, estos resultados avalan la eficacia de la mecánica de puntos y premios en estrategias de gamificación en educación como lo indican Simões, Redondo y Vilas (2013), Hanson-Smith (2016), y Dicheva (2015). Además, incorporar las TIC como una interacción, en la estrategia de gamificación, se observó limitaciones en los estudiantes, que se espera ir corrigiendo por el beneficio de la comunidad universitaria.

En consecuencia, se valida la efectividad de la mecánica gamificación en educación usando puntos y premios documentada por autores como Zichermann y Cunningham (2011); Lee y Hammer (2011); Simões, Redondo y Vilas (2013); Dicheva (2015); Iosup y Epema (2014).

\section{CONCLUSIONES}

Para mejorar la educación en las universidades es necesario utilizar herramientas pertinentes que motiven a desarrollar contenidos, participaciones. El éxito de una estrategia de gamificación en educación superior radica en el diseño, el modelo de Werbach y Hunter (2012) resulta conveniente y práctico en la medida en que establece un marco general de planeación, implementación $y$ seguimiento.

Se validó la efectividad de la estrategia de gamificación en educación usando puntos y premios; sin embargo, es indispensable para mejorar la experiencia de juego incluir las recomendaciones de los estudiantes y algo que para ellos es fundamental es no hacer examen final. 


\section{REFERENCIAS}

Briones, G. (2002). Metodología de la investigación cuantitativa en las ciencias sociales. Bogotá: ARFO editores.

Burke, B. (2012). Gamificación 2020: ¿Cuál es el futuro de la gamificación? Gartner. Recuperado de https://www.gartner.com/doc/2226015/ Gamificatio zn--future-zGamification

ClassDojo. (2017, noviembre 7). Acerca de nosotros. Recuperado 7 de noviembre de 2017, de https://www.classdojo.com/es-MX/about

Deterding, S. (2011). Gamification: designing for motivation. Interactions, New York. 19(4), 14-17.

Deterding, S.; Khaled, R.; Nacke, L. y Dixon, D. (2011). Gamification: toward a definition. Actas del taller de gamificación de CHI 2011, Vancouver, 2011, 12-15. Disponible en http:// gamification-research.org/wp-content/ uploads/2011/04/02-Deterding-KhaledNacke-Dixon.pdf

Dicheva, D. (2015). Gamification in Education: A Systematic Mapping Study. Educational Technology \& Society, 18(3), 75-88.

Escribano, F. (2013). Gamificación versus Ludictadura. Obra Digital, (5), 58-72.

Fernández, A.; Olmos, J. y Alegre, J. (2016). Pedagogical value of a common knowledge repository for business management courses. @ Tic: Revista d’Innovació Educativa, (16), 39-47.

Hanson-Smith, E. (2016). Games, gaming, and gamification: Some aspects of motivation. Tesol Journal, 7(1), 227-232.
Iosup, A., y Epema, D. (2014, March). An experience report on using gamification in technical higher education. In Proceedings of the 45th ACM technical symposium on Computer science education (pp. 27-32).

Kapp, K. (2012). Games, Gamification, and the quest for learner engagement. Training and Development, 66(6), 64-68.

Lee, J. y Hammer, J. (2011). Gamification in Education: What, How, Why Bother? Academic Exchange Quarterly, 15(2),1-5.

Marín, V. (2015). La gamificación educativa: una alternativa para la enseñanza creativa. Digital Education Journal, (90), 1-4.

Prensky, M. (2003). Digital natives, digital immigrantsA new way to look at ourselves and our kids. Recuperado de http:// www. marcprensky.com/writing/ Prensky\%20

Ramírez, J. (2014). Gamificación: mecánicas de juegos en tu vida personal y profesional. México, México: Alfaomega Grupo Editor. Recuperado de https:// unisabana22.gsl.com.mx/exlibris/aleph/ u22_1_cna/objects/cna01/view/ 19/146705_000076290.jpg.

Simões, J., Redondo, R.y Vilas, A. (2013). A social gamification framework for a K-6 learning platform. Computers in Human Behavior, 29(2), 345-353. https://doi.org/10.1016/ j.chb.2012.06.007

Valderrama, B. (2015). Los secretos de la gamificación: 10 motivos para jugar. Capital Humano, 
$\begin{array}{llllllllllll}R & E & V & I & S & T & A\end{array}$

P u bl i c a n d o

I S S N $13990-9304$

(295), 73-78.

Werbach, K.y Hunter, D. (2012). For the Win: How

Game Thinking Can Revolutionize Your

Business. Philadelphia: Wharton Digital

Press.

Zichermann, G.y Cunningham, C. (2011).

Gamification by Design: Implementing

Game Mechanics in Web and Mobile Apps.

Sebastopol, California: O'Reilly. 